\title{
Viscosity Reduction of Heavy Oil by Aquathermolysis with Coordination Complex at Low Temperature
}

\author{
Hua-rui Hao ${ }^{1}$, Hui-jun $\mathrm{Su}^{1}$, Gang Chen ${ }^{*}, 2$, Jing-rui Zhao ${ }^{3}$ and $\mathrm{Li} \mathrm{Hong}^{4}$ \\ ${ }^{I}$ School of Chemistry and Chemical Engineering, Yulin University, Yulin 719000, China \\ ${ }^{2}$ College of Chemistry and Chemical Engineering, Xi'an Shiyou University, Xi'an, 710065, China \\ ${ }^{3}$ Shaanxi Yanchang Petroleum Oilfield Chemical Technology Co., LTD, Yan'an, 717400, China \\ ${ }^{4}$ Jiangsu Santacc Co., Ltd. Zhengjiang, 212200, China
}

\begin{abstract}
To seek for new catalysts for the aquathermolysis of crude oil under relatively low temperature, in this paper, a series of Mannich base-transit metal coordination complexes was synthesized and used in aquathermolysis of heavy oil as catalyst. The results show an evident viscosity reduction by $84.2 \%$ for the heavy oil reacted at $180{ }^{\circ} \mathrm{C}$ for $24 \mathrm{~h}$ catalyzed by the $\mathrm{Ni}(\mathrm{II})$ coordination complex. The group compositions, the element content of the heavy oil and paraffin crystal morphology before and after the aquathermolysis reaction were analyzed, which are consistent with the viscosity reduction of the heavy oil. Besides the catalysts were also found to be widely applicable.
\end{abstract}

Keywords: Aquathermolysis, catalyst, coordination complex, heavy oil, viscosity reduction.

\section{INTRODUCTION}

The reserves of crude oil account for more than $70 \%$ of the total quantity of petroleum all over the world. However, due to the high viscosity and density, it is very difficult to handle heavy crude oils. Therefore, many researchers are devoting to reduce viscosity of heavy oil for the production and transportation $[1,2]$. It was reported that metals can catalyze the aquathermolysis, and thereafter the uses of catalysts in this reaction have been screened [3]. Now, the catalytic aquathermolysis has been applied to solve the viscosity problem during exploration of heavy crude oil. The research has proved that superheated water $\left(240-300^{\circ} \mathrm{C}\right)$ can heat the hydrocarbon leading asphaltene and resin molecules to break down to small molecules, therefore the viscosity is reduced $[4,5]$, and if some catalyst is employed in this reaction, the viscosity can be reduced deeply. Commonly, after aquathermolysis, the amount of saturate and aromatics will increase, while the amount of asphaltene and resin will decrease [6-9]. Generally, the catalysts for aquathermolysis include mineral, water soluble, oil soluble, and dispersed catalysts. In fact, the oil soluble catalysts and dispersed catalysts are more active than the water-soluble catalyst. But the cost of the two former types of catalysts is much higher than that of simple water soluble catalysts. Hence, more research is focusing on making this process more economical. Another problem is the reaction condition of these catalysts when used in the oil field. The activity of the catalysts is affected by the temperature obviously. In the indoor experiments, the temperature can be controlled under a certain temperature, but during the oil field application

*Address correspondence to this author at the College of Chemistry and Chemical Engineering, Xi'an Shiyou University, Xi'an, 710065, China; Tel/Fax: 086-029-88382693; E-mail: gangchen@xsyu.edu.cn
, when the superheated water is injected into the oil reservoir, the oil surface temperature is higher, however, temperature is gradually lower on the depth of the oil floor, and hence the catalyst loses its activity. So, further research should address the catalysts' activity under low temperature. Several groups have investigated the heavy oil aquathermolysis catalyzed by water-soluble catalysts under relative low temperature $\left(160-280^{\circ} \mathrm{C}, 10-25 \mathrm{MPa}, 24-240 \mathrm{~h}\right)$, but the viscosity reduction is only about $60 \%[9,10]$.

In our previous work, a series of transition metal coordination complex was prepared from $p$-chlorophenol Mannich base and transition metal chloride and applied as catalyst in the aquathermolysis of heavy oil at relatively low temperature. In this wok, the most effective one can reduce the viscosity by more than $70 \%$ under the relatively low reaction temperature of $180{ }^{\circ} \mathrm{C}$ [11]. But $p$-chlorophenol is poisonous for human beings during the preparation and application. To seek more human-friendly and effective catalyst for the aquathermolysis of heavy oil, a series of Mannich base-transition metal complex was prepared and evaluated.

\section{EXPERIMENTAL}

\subsection{Materials}

Petroleum ether, toluene, ethanol, formaldehyde, $p$ methylphenol, and piperazine were purchased from Tianjin Kermel Chemical Reagent Co., Ltd (at AR reagent grade) and were used without further purification. The heavy oil samples (1\#, 2\#, and 3\#) used for evaluations were obtained from Yumen Oilfield, whose properties are shown in Table $\mathbf{1}$. 
Table 1. The physical parameters of heavy oil from Yumen oil field.

\begin{tabular}{|c|c|c|c|c|c|c|c|}
\hline Oil Sample & $\boldsymbol{\eta}^{\mathbf{5 0}} \mathbf{( \mathbf { m P a } \cdot \mathbf { s } )}$ & Pour Point $\left.\mathbf{(}^{\mathbf{0}} \mathbf{C}\right)$ & $\boldsymbol{\rho}^{\mathbf{2 0}} \mathbf{( \mathbf { g } \cdot \mathbf { c m } ^ { - \mathbf { 3 } } )}$ & $\begin{array}{c}\text { Saturated HC } \\
\mathbf{( \% )}\end{array}$ & $\begin{array}{c}\text { Aromatic HC } \\
\mathbf{( \% )}\end{array}$ & $\begin{array}{c}\text { Resin }(\mathbf{\%}) \\
\mathbf{( \% )}\end{array}$ \\
\hline \hline $1 \#$ & 12500 & 41.2 & 0.901 & 51.83 & 9.66 & 23.92 & 14.6 \\
\hline $2 \#$ & 25530 & 46.5 & 0.907 & 48.37 & 13.55 & 25.50 & 12.58 \\
\hline $3 \#$ & 30510 & 51.2 & 0.912 & 50.87 & 5.58 & 19.95 & 23.60 \\
\hline
\end{tabular}

2.2. Synthesis and Characterization of the Complexes [12, 13]

The complexes were synthesized as shown in Fig. (1) and the corresponding names are listed in Table 2. A mount of $p$ methylphenol $(2 \mathrm{mmol})$, formaldehyde $(2.2 \mathrm{mmol})$ and piperazine $(1 \mathrm{mmol})$ was dissolved in ethanol and refluxed for $4 \mathrm{~h}$ under stirring. A methanol solution of $\mathrm{MCl}_{\mathrm{n}}(1 \mathrm{mmol})$ was added dropwise and stirred for another $2 \mathrm{~h}$. The mixture was cooled to room temperature and filtrated, the solution was kept at room temperature for $7 \mathrm{~d}$, and the natural evaporation gave crystals for the further use. The IR spectra of a selected complex were recorded in $\mathrm{KBr}$ pellets with a Bruker Tensor 37 spectrometer in the $400-4,000 \mathrm{~cm}^{-1}$ region.

Table 2. The composition and the name of the complexes.

\begin{tabular}{|c|c|c|c|c|c|}
\hline $\mathbf{M}$ & $\mathbf{F e}($ III) & $\mathbf{C o}($ II) & $\mathbf{N i ( I I )}$ & $\mathbf{C u}($ II) & $\mathbf{Z n ( I I )}$ \\
\hline \hline Name & MPC-1 & MPC-2 & MPC-3 & MPC-4 & MPC-5 \\
\hline
\end{tabular}

\subsection{Catalytic Aquathermolysis Reaction of the Heavy Oil by the Complexes [13-15]}

All the experiments were carried out by adding heavy oil, water and catalyst in a designed mass ratio of the crude oil quality into a reactor. Then the reactants were heated to 180 'C for aquathermolysis reaction for $24 \mathrm{~h}$. After the reaction, the mixture was cooled to about $50^{\circ} \mathrm{C}$ in a water bath and dumped into a baker, thereby the oil sample was obtained by decant the water for test and analysis. The reaction conditions and catalysts screening were carried out using 1\# heavy oil.

The viscosity of the heavy oil was evaluated by a
BROOKFIELD DV-II Viscometer at different temperature. The viscosity reduction rate $\Delta \eta \%$ was calculated as follows: $\Delta \eta \%=\left(\left(\eta_{0}-\eta\right) / \eta_{0}\right) \times 100$, where $\Delta \eta(\%)$ is the viscosity reduction rate, $\eta 0(\mathrm{mPa} \cdot \mathrm{s})$ is the viscosity of the oil before the reaction, and $\eta(\mathrm{mPa} \cdot \mathrm{s})$ is the viscosity of the oil after the reaction. Three readings were made on each sample to check repeatability and to obtain an average value and the relative standard deviation was within $2.0 \%$. The pour point was tested according to the ASTM-97 procedure. The initial temperature is set at $2.5{ }^{\circ} \mathrm{C}$ above the temperature at which the oil became solid and the detection limit is $0.1{ }^{\circ} \mathrm{C}$ [14]. Three readings were made on each sample to check repeatability and to obtain an average value and the relative standard deviation was within $2.0 \%$, and the changed pour point $(\Delta \mathrm{P})$ was calculated as follows: $\Delta \mathrm{P}=\mathrm{P}-\mathrm{P}_{0}$. Furthermore, the structural changes and group compositions of the heavy oil before and after reaction were further analyzed. The four composition groups, asphaltenes, resins, saturated hydrocarbons and aromatic hydrocarbons were separated by the column chromatography-based SARA method according to the standard of China Petroleum SY/T 5119 [15]. Elemental analysis of the heavy oil before and after reaction was performed on an elemental analyzer (vario EL). The paraffin crystal in the saturated HC of the heavy oil before and after aquathermolysis was investigated using a BX41-P OLYMPUS polarizing microscope under $15^{\circ} \mathrm{C}$.

\section{RESULTS AND DISCUSSION}

\subsection{Characterization of the Catalyst}

In order to study the binding mode of the Mannich base to the metal ion in the complexes, the IR spectrum of the free ligand (Mannich base) was compared with the spectra of the Ni(II) complex, as shown in Fig. (2). The IR spectra of the<smiles>C[C+](C)O[C@H]1CNCCN1</smiles>

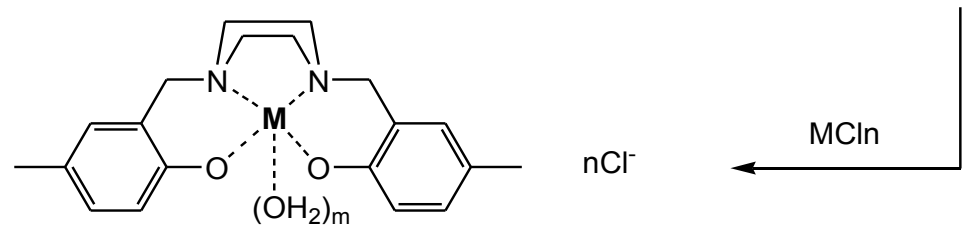

$\mathrm{M}=\mathrm{Fe}(\mathrm{III}), \mathrm{Co}(\mathrm{II}), \mathrm{Ni}(\mathrm{II}), \mathrm{Cu}(\mathrm{II}), \mathrm{Zn}(\mathrm{II})$

Fig. (1). Preparation of p-methyl-phenol Mannich base-transition metal complexes. 
complex show a wide and deep band in the region of 3300 $\mathrm{cm}^{-1}$, which are assigned to coordination $\mathrm{H}_{2} \mathrm{O}$ of the complex. The bands in lingand spectra due to $\mathrm{n}(\mathrm{C}-\mathrm{O})$ is located in the regions $1130 \mathrm{~cm}^{-1}$. In the spectra of complex, the $\mathrm{n}(\mathrm{C}-\mathrm{O})$ mode of the free ligand moves to $1086 \mathrm{~cm}^{-1}$ indicating the enolization of $\mathrm{C}-\mathrm{O}$ followed by deprotonation and complexation with metal ions. The bands in the region of $1599 \mathrm{~cm}^{-1}$ and $1499 \mathrm{~cm}^{-1}$, which are assigned to $\mathrm{n}(\mathrm{Ph})$ of the ligand, which move to $1614 \mathrm{~cm}^{-1}$ and $1513 \mathrm{~cm}^{-1}$ respectively in the complex. It is concluded that the ligand acts as a monobasic tetra-dentate manner in the complex [16].

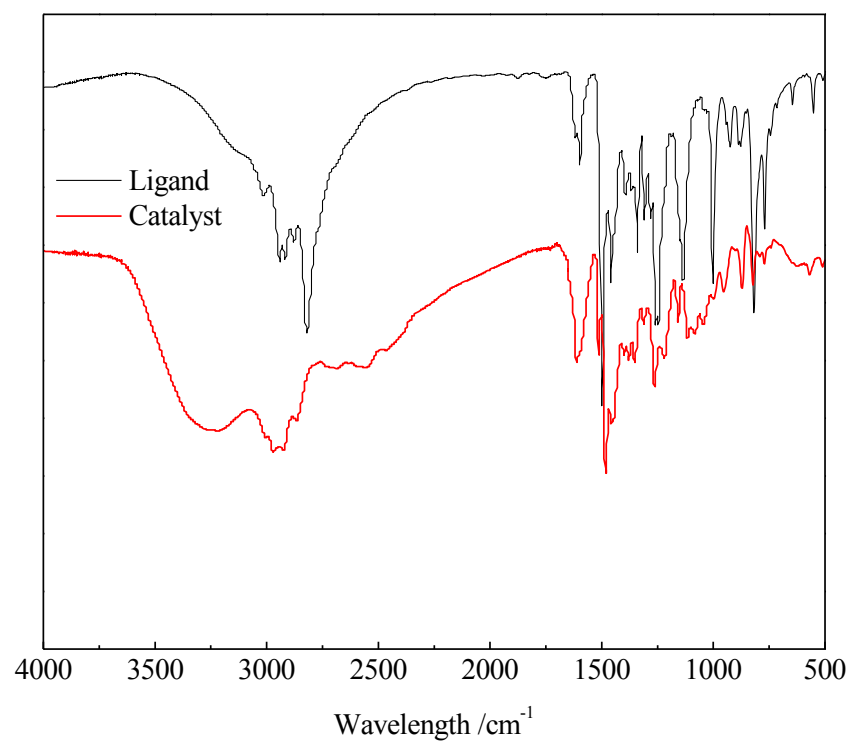

Fig. (2). The IR spectrum spectra of the ligand and the catalyst$\mathrm{Ni}(\mathrm{II})$ complex.

\subsection{Aquathermolysis Reaction Condition Screening}

From the beginning, 1\# heavy oil was used for the reaction conditions and catalyst screening. The effect of mass ratio of water to oil on the viscosity in the reaction was investigated, under the settled temperature and reaction time, $180{ }^{\circ} \mathrm{C}$ and $24 \mathrm{~h}$, and the results are summarized in Fig. (3). It is quite clear that water addition to the heavy oil can help to reduce the viscosity, depending on the mass ratio. This decreased tendency is quite clear as the water content increases before the mass ratio of water to oil reaches to 0.3 . With the ratio varying from 0.3 to 0.5 , the viscosity reduction is almost in the same level, and when the mass ratio reaches 0.6 , it presents a slightly ascending tendency. So the mass ratio of water to oil was settled as 0.3 in the following experiments [11].

\subsection{Catalyst Screening}

This series of coordination complex was used in the aquathermolysis reaction as catalyst with the concentration of $0.5 \%$, and the results are summarized in Table 3 (the viscosity was tested at $50^{\circ} \mathrm{C}$ ). As shown in this table, not all the complexes are effective in this reaction, only MMC-1, MMC-3 and MMC-5 can catalyze the reaction, among which MMC-3 is the most effective one with the $84.2 \%$ viscosity reduction rate and $10.3{ }^{\circ} \mathrm{C}$ pour point depression. On the contrary, MMC-2 and MMC-4 cannot help to reduce the viscosity and pour point, but lead to some increase. Therefore, MMC-3 (Ni(II) complex) was selected as the catalyst in the further study.

Table 3. The aquathermolysis reaction results using coordination complexes as catalysts.

\begin{tabular}{|c|c|c|}
\hline Catalyst & $\boldsymbol{\Delta \eta}(\mathbf{\%})$ & $\Delta \mathbf{P}\left({ }^{\circ} \mathbf{C}\right)$ \\
\hline \hline MMC-1 & 33.4 & 4.2 \\
\hline MMC-2 & -8.5 & -0.8 \\
\hline MMC-3 & 84.2 & 10.3 \\
\hline MMC-4 & -8.3 & -1.2 \\
\hline MMC-5 & 12.5 & 2.3 \\
\hline
\end{tabular}

\subsection{Effect of Catalyst Concentration on the Aquathermolysis Reaction}

The catalyst plays the most important role in this reaction, so the effect of catalyst concentration on the aquathermolysis reaction was investigated in the following study. Fig. (4) shows that the viscosity reduction efficiency depends on the catalyst concentration. The viscosity keeps dropping as the concentration increases from $0.1 \%$ to $0.5 \%$ with the viscosity dropping from $18700 \mathrm{mPa} \cdot \mathrm{s}$ to 3720 $\mathrm{mPa} \cdot \mathrm{s}$, and it dose not change obviously as the concentration

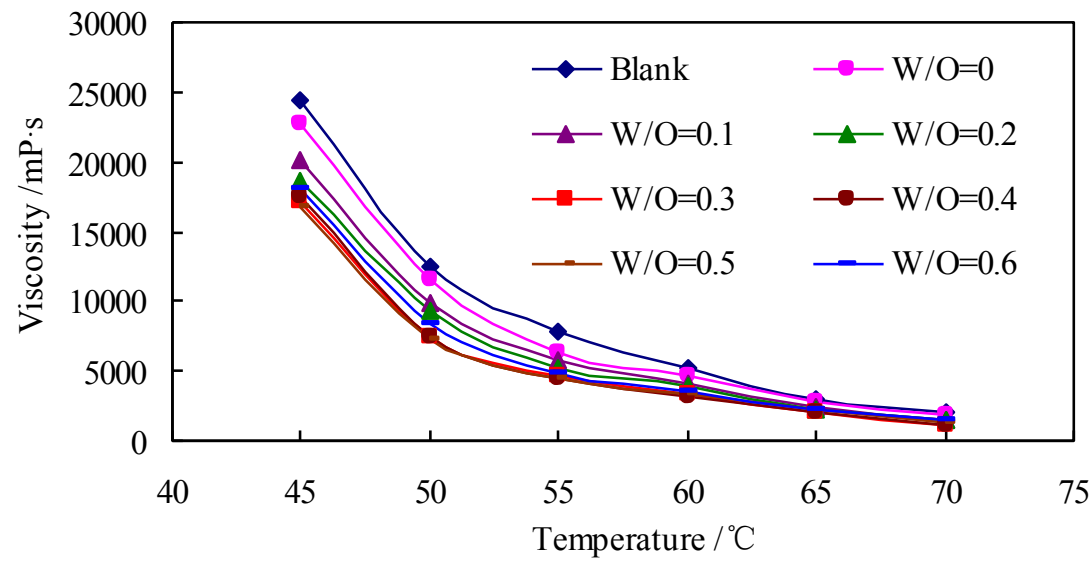

Fig. (3). The influence of water on the viscosity of crude oil after aquathermolysis reaction. 


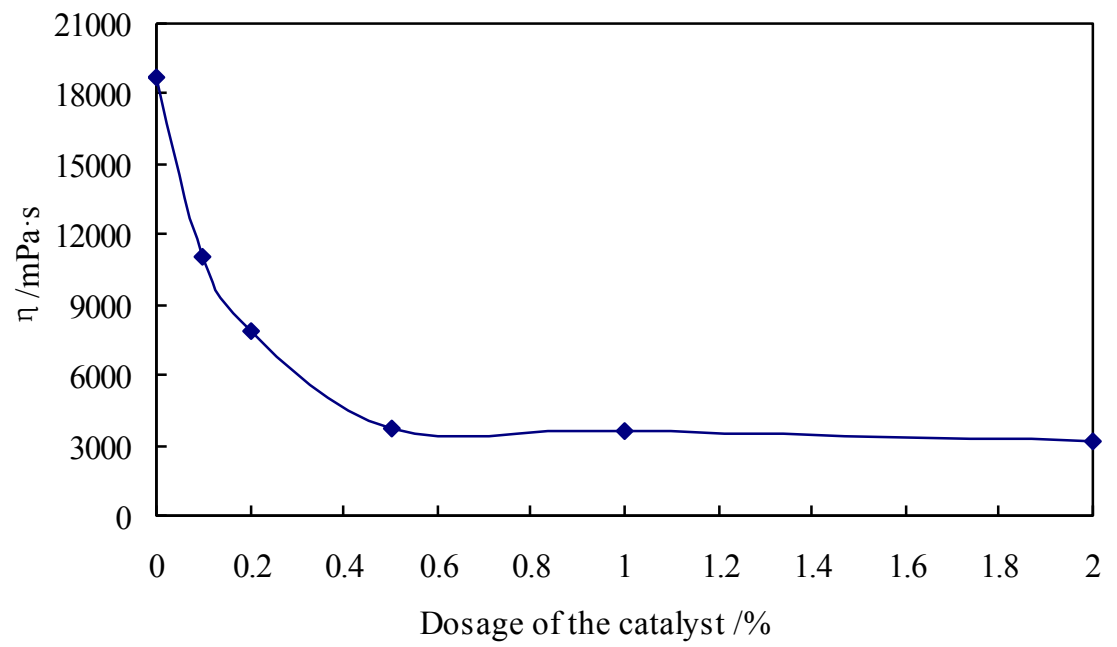

Fig. (4). Effect of catalyst concentration on the viscosity.

beyond $0.5 \%$, so the optimum concentration should be $0.5 \%$.

\subsection{Group Compositions Analysis}

Theoretically, there is a chemical reaction during the process, so the compositions should change to some degree. The contents of the group compositions of heavy oil before and after reaction are listed in Table 4 . It can be found that the saturate $\mathrm{HC}$ and aromatic $\mathrm{HC}$ increase, as resin and asphaltene decrease after reaction. The original SARA compositions of feedstock are $51.83 \%, 9.66 \%, 23.92 \%$ and $14.59 \%$, respectively. When MMC-3 and water are in coexistence in heavy oil, the saturate increased to $55.75 \%$, aromatic increased to $11.67 \%$, resin decreased to $21.25 \%$ and asphaltene decreased to $11.33 \%$ after reation. It reveals that the reduced part of resin and asphaltene depolymerized to aromatic and saturated $\mathrm{HC}$.

\subsection{Paraffin Crystal Morphology Study}

As shown in Table 5, the saturated HC content increases after aquathermolysis, which can improve the fluid ability of the crude oil. The change of the compositions will lead to the change of the status under low temperature, so the morphology of paraffin crystal in saturated HC was investigated, and the photographs are shown in Fig. (5). From the figure, the paraffin crystals with larger size in the saturated $\mathrm{HC}$ are found before aquathermolysis (left). For the sample after aquathermolysis (right), the paraffin crystals are smaller and less than the left one, which indicates that the aquathermolysis can reduce the tendency of crystals to form three-dimensional network, so as to reduce the viscosity and depress the pour point of the heavy oil [20].

Table 4. Group compositions of heavy oil before and after reaction.

\begin{tabular}{|c|c|c|c|c|}
\hline Oil Sample & Saturated HC (\%) & Aromatic HC (\%) & Resin (\%) & Asphaltene (\%) \\
\hline \hline before reaction & 51.83 & 9.66 & 23.92 & 14.59 \\
\hline after reaction & 55.75 & 11.67 & 21.25 & 11.33 \\
\hline
\end{tabular}

\subsection{Element Analysis}

The change of the compositions can surely cause the change of element ratio in the oil. Element analysis results of oil samples are listed in Table 5. It was found that the amount of heteroatoms in oil samples is $8.3 \%$ before reaction, which reveals that many free radicals can generate during the aquathermolysis by the cleavage of $C-R(R=N$, $\mathrm{O}, \mathrm{S})$ bonds [17]. After reaction, the contents of $\mathrm{C}$ and $\mathrm{H}$ increase, and the contents of $\mathrm{N}, \mathrm{S}$ and other elements are depressed to $7.20 \%$ totally. It can be attributed to the cleavage of C-R to produce some water-soluble compounds, such as some small molecular amine, alcohol and sulphide $[18,19]$.
Table 5. Element content of oil sample before and after reaction.

\begin{tabular}{|c|c|c|c|c|c|}
\hline \multirow{2}{*}{ Oil Sample } & \multicolumn{5}{|c|}{ Composition (\%) } \\
\cline { 2 - 6 } & $\mathbf{C}$ & $\mathbf{H}$ & $\mathbf{N}$ & $\mathbf{S}$ & Other \\
\hline \hline before reaction & 81.31 & 10.39 & 0.45 & 0.42 & 7.43 \\
\hline after reaction & 82.25 & 10.55 & 0.25 & 0.33 & 6.62 \\
\hline
\end{tabular}

\subsection{Application in Other Heavy Oils}

As the reaction conditions and catalyst have been screened with using $1 \#$ heavy oil, other heavy oil samples 

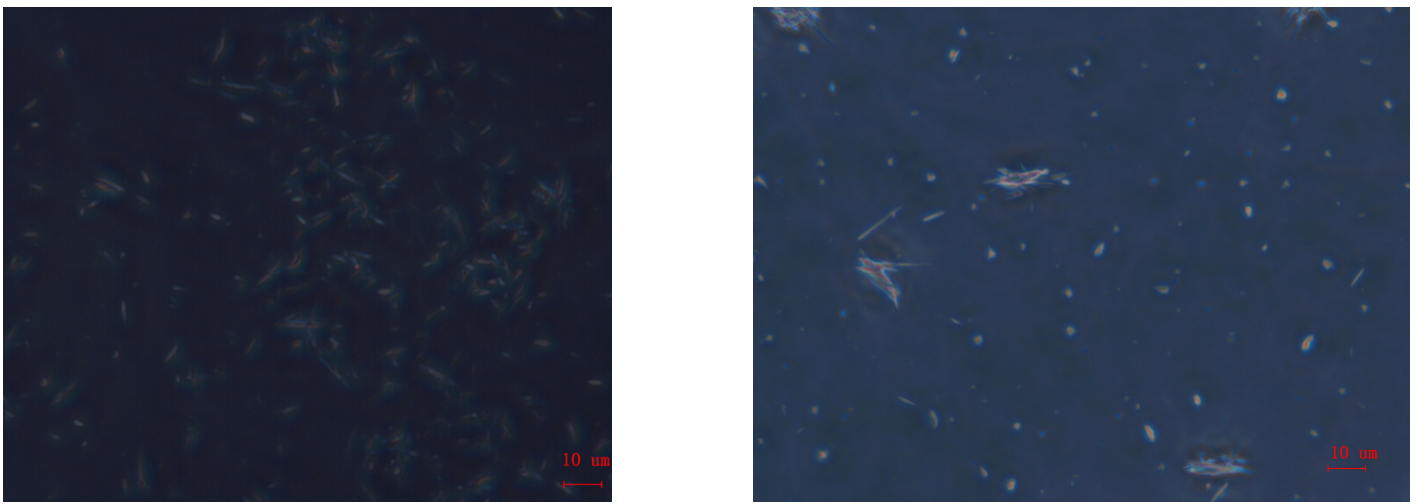

Fig. (5). Photography of paraffin crystal in saturated $\mathrm{HC}$ of the 1 \# heavy oil before (left) and after (right) aquathermolysis.

Table 6. The aquathermolysis results of $2 \#$ and $3 \#$ heavy oil.

\begin{tabular}{|c|c|c|c|c|c|c|c|c|}
\hline \multirow{2}{*}{ Oil Sample } & \multirow{2}{*}{ Catalyst } & \multicolumn{9}{|c|}{ Viscosity (mPa·s) } \\
\cline { 3 - 9 } & & $\mathbf{4 0}{ }^{\mathbf{0}} \mathbf{C}$ & $\mathbf{4 5}{ }^{\circ} \mathbf{C}$ & $\mathbf{5 0}{ }^{\circ} \mathbf{C}$ & $\mathbf{5 5}^{\mathbf{}} \mathbf{C}$ & $\mathbf{6 0}^{\mathbf{0}} \mathbf{C}$ & $\mathbf{6 5}^{\circ} \mathbf{C}$ & $\mathbf{7 0}^{\mathbf{}} \mathbf{C}$ \\
\hline \hline $2 \#$ & - & 67322 & 43900 & 25530 & 13577 & 7820 & 5350 & 3455 \\
\hline $2 \#$ & MMC-3 & 24335 & 12597 & 6003 & 3875 & 2550 & 2235 & 2010 \\
\hline $3 \#$ & - & 85070 & 60303 & 30510 & 18560 & 9020 & 6155 & 3896 \\
\hline $3 \#$ & MMC-3 & 17902 & 11580 & 5859 & 3776 & 2011 & 1977 & 1884 \\
\hline
\end{tabular}

were investigated in such conditions with $0.5 \% \mathrm{MMC}-3$, and the results are shown in Table 6. From the results, it can be found that the viscosity of the two heavy oil samples can be decreased effectively by $70-80 \%$ at lower temperature, which means the reaction conditions and the catalyst are widely applicable.

\section{CONCLUSION}

In this work, a series of Mannich base-transit metal coordination complexes was synthesized and used in the catalytic aquathermolysis of heavy oil. Under the optimized reaction condition at $180{ }^{\circ} \mathrm{C}$ for $24 \mathrm{~h}$ catalyzed by the $\mathrm{Ni}$ (II) coordination complex, the viscosity of heavy oil was reduced by $84.2 \%$. With the catalysis, the asphaltene and resin were partly pyrolyzed into small molecules, and the heteroatom contents were also decreased. All of these changes were unregressed, and finally led to the viscosity reduction. This type of coordination complex is widely applicable and has great potential for further applications in catalytic aquathermolysis of heavy.

\section{CONFLICT OF INTEREST}

The authors confirm that this article content has no conflict of interest.

\section{ACKNOWLEDGEMENTS}

This work was financially supported by the grants from National Science Foundation of China (21376189), Scientific and Technological Plan Projects of Shaanxi Province (2014TG-09) and Scientific Research Program Funded by Shaanxi Provincial Education Department (2013JK0647).

\section{REFERENCES}

[1] Hu, C.Z. Heavy oil exploitation technology; Petroleum Industry Publishing Inc.: Beijing, 1998

[2] Lu, L.H.; Li, M.H.; Su, Y.L. The overview of the heavy oil exploitation. Neimenggu Chemistry Industry, 2005, 3, 110-112.

[3] Hyne, J.B.; Greidanus, J.W. Aquathermolysis of heavy oil. In: Proceedings of second international conference on heavy crude and tar sands, Caracas Venezuela, 1982, pp. 25-30.

[4] Clark, P.D.; Hyne, J.B. Steam-oil chemical reactions: mechanisms for the aquathermolysis of heavy oil. AOSTRA J. Res., 1984, 1: 1520.

[5] Hyne, J.B. A synopsis of work on the chemical reactions between water and heavy oil sands during stimulated steam stimulation. AOSTRA Synopsis Report No. 50, Aquathermolysis, AOSTRA Contracts No.11103103B/C, 1986

[6] Maity, S.K.; Ancheyta, J.; Marroquín, G. Catalytic aquathermolysis used for viscosity reduction of heavy crude oils: a review. Energy Fuels, 2010, 24, 2809-2816.

[7] Yousef, H.S.; Tayfun, B. Viscosity reduction of heavyoil/bitumen using micro- and nano-metal particles during aqueousand nonaqueous thermal applications. J. Polym. Eng., 2014, (119), 210220.

[8] Yang, X.; Gates, I.D. Design of hybrid steam-in situ combustion bitumen recovery Processes. Nat. Resources Res.. 2009, 18(3), 213233.

[9] Zhong, L.G.; Liu, Y.J.; Fan, H.F. In: Jiang, S.J. Liaohe ExtraHeavy Crude Oil Underground Aquathermodytic Treatments Using Catalyst and Hydrogen Donors Under Steam Injection Conditions. SPE International Improved Oil Recovery Conference in Asia Pacific, Kuala Lumpur, Malaysia, October 20-21, 2003; Paper No. 84863-MS.

[10] Clark, P.D.; Dowling, N.I.; Hyne, J.B.; Lesage, K.L. The chemistry of organosulphur compound types occurring in heavy oils: 4 . The high-temperature reaction of thiophene and tetrahydrothiophene with aqueous solutions of aluminium and first-row transition-metal cations. Fuel, 1987, 66, 1353-1357.

[11] Zhang, J.; Li, X.L.; Chen, G.; Su, H.J.; Zhao, W. Study on aquathermolysis of heavy oil at relatively low temperature catalyzed by water-soluble complexes. J. Fuel Chem. Technol., 2014, 42(4), 443-448.

[12] Chen, G..; Tang, Y.; Gu, X.F.; Zhang, J. Synthesis structure and catalysis study on degradation reaction of PAM of 2-(N,N- 
biscarboxymethyl) aminomethyl-4- chloro-phenol $\mathrm{Cu}(\mathrm{II})$. Chem. Res. Appl., 2011, 23(3), 268-272.

[13] Gu, X.F.; Qin, F.L.; Chen, G. Synthesis and Catalysis Study of Fenton-like system Catalyst on Degradation Reaction of PAM. Environ. Pollut. Control, 2012, 34(12), 13-16.

[14] Analysis of family composition of rock extract and crude oil by TLC-FID. China National Petroleum Corporation, 1997.

[15] Analysis of family composition of rock extract and crude oil by column chromatography. China National Petroleum Corporation, 1995.

[16] Shanmuga, B.K.; Sreedaran, S.; Kalilur, R.A.; Rajesh, K.; Narayanan, V. Synthesis, spectral, magnetic, electrochemical and kinetic studies of copper(II), nickel(II) and zinc(II) acetate complexes derived from phenol based "end-off" ligands: Effect of P-substituents. Polyhedron, 2007, 26(14), 3993-4002.

[17] Chao, K.; Chen, Y.L.; Liu, H.C.; Zhang, X.M.; Li, J. Laboratory experiments and field test of a difunctional catalyst for catalytic aquathermolysis of heavy oil. Energy Fuels, 2012, 26(2), 11521159.

[18] Wang, Y.Q. Thesis, China University of Geosciences, 2010

[19] Fan, H.F.; Zhang, Y.; Lin, Y.J. The catalytic effects of minerals on aquathermolysis of heavy oils. Fuel, 2009, 83, 2035-2039.

[20] Chen, G.; Tang, Y.; Zhang, J. Synthesis and application of polyaminoamide as paraffin inhibitor from vegetable oil. Chem. Central J., 2011, 5, 82-86.

(C) Hao et al.; Licensee Bentham Open.

This is an open access article licensed under the terms of the Creative Commons Attribution Non-Commercial License (http://creativecommons.org/licenses/by-nc/3.0/) which permits unrestricted, non-commercial use, distribution and reproduction in any medium, provided the work is properly cited. 